\title{
NOVEL COMPACT DUAL-BROADBAND PLANAR METAMATERIAL ANTENNA
}

\author{
Dang Nhu Dinh ${ }^{1,2, *}$, Dinh Thanh Liem¹, Huynh Nguyen Bao Phuong3, \\ Hoang Phuong Chi ${ }^{1}$, Dao Ngoc Chien ${ }^{4}$ \\ ${ }^{1}$ Hanoi University of Science and Technology, 01 Dai Co Viet, Hanoi, Viet Nam \\ ${ }^{2}$ The University of Fire Fighting and Prevention, 243 Khuat Duy Tien, Hanoi, Viet Nam \\ ${ }^{3}$ Quy Nhon University, 170 An Duong Vuong, Binh Binh, Viet Nam \\ ${ }^{4}$ Ministry of Science and Technology, 113 Tran Duy Hung, Hanoi, Viet Nam
}

"Email: dinh.dangnhu-set@hust.edu.vn

Received: 25 July 2016, Accepted for publication: 15 January 2017

\begin{abstract}
This paper proposes a novel uni-planar dual-band antenna using Composite Right Left Handed (CRLH) transmission line (CRLH-TL). Proposed antenna is designed based on the fringing effects of metamaterials and combined with coplanar waveguide (CPW) feeding in order to create two frequency bands for WLAN applications at the 2.4 and $5.5 \mathrm{GHz}$ bands. Principle of gradual transform is applied to the antenna for extending the resonance frequency ranges. Optimized metamaterial antenna are fabricated and measured. Measurement results showed that the antenna operates in two broad frequency ranges spreading from 1.8 to $3.62 \mathrm{GHz}$ and from 4.85 to $7.52 \mathrm{GHz}$ with very compact overall dimensions of $18 \mathrm{~mm} \times 16 \mathrm{~mm}\left(0.147 \lambda_{0}\right.$ $\left.\times 0.13 \lambda_{0}\right)$.
\end{abstract}

Keywords: metamaterial transmission line, monopole antenna, fringing effect.

\section{INTRODUCTION}

Recently, wireless communication systems have rapidly growing with the requirements of compact electronic devices. Therefore, the antenna device also must be small in size, lightweight and easy to fabricate. Normally, antenna size is always inversely proportional to its operating frequency. Such that, the size of the antenna will be enlarged at low frequency bands. For what concerns of tackling this issue, there have been many techniques proposed to reduce the size of antennas [1 - 10]. The transmission line metamaterials (TL-MM) [1] have been employed to construct small resonant antennas [2, 3, 10, 11]. In [11], a metamaterial loading with metallic bridges was used to create a second resonant mode, which is lower than the first resonant mode created by the conventional patch. In [3], a monopole antenna was proposed based on composite right/left handed (CRLH) unit cell with metallic vias. This proposed antenna can be used in 
GSM-900/WLAN/LTE-2500 applications. It is obvious that the participation of metallic bridge or vias will make the difficulty in fabrication and impact to the exactly of the experimental measurements.

In [8], a dual-band CRLH antenna was proposed by etching a complementary split ring resonator (CSRR) on the surface of the patch to resonate at higher frequencies, while resonance at low frequency range was created by the T-shaped slot cut on the ground plane. Similarly, a coplanar waveguide (CPW) antenna was also designed by etching a CSRR structure on the patch, however, in order to resonate at lower frequency and slits on the coplanar ground plane for resonating at the high frequency band [9]. On the other hand, two inter-digital structures were implemented on the patch to formed dual band antenna [12]. In addition, CPW-fed antennas are designed based on metamaterial-inspired loading to achieve the compactness in size $[7,13,14]$. These antennas are designed in single-layered, vialess and can be easily fabricated at a low cost but their overall size are quite large. Moreover, slit rings are etched on the metallic surface of patch to construct the compact antennas [15 - 17]. These split rings introduce the higher resonant modes that are suitable for the multi-band antennas.

With the aim of overcoming aforementioned drawbacks, in this paper, a monopole antenna is proposed by employing CRLH-TL and fringing effects of metamaterial to reduce overall size and to create dual operation bands. The gradual transform is used to enlarge two frequency bands of the antenna. In proposed design, the vias in the CRLH model are replaced by meandered lines locating on top surface of the proposed antenna. The antenna has been fabricated and measured. Measured S-parameters are given and compared with simulated results, showing a great agreement.

\section{DUAL-BROADBAND METAMATERIAL ANTENNA DESIGN}

\subsection{Configuration of proposed antenna}

The proposed antenna is a monopole antenna fed by coplanar waveguide. The antenna is printed on FR4 dielectric substrate with a dielectric constant $\varepsilon_{r}=4.4$ and thickness $h=0.8 \mathrm{~mm}$. The configuration of proposed antenna shown in Figure 1 consists of three parts: the first is the radiation patch placed at the center of the antenna. In this design, the rectangular patch in conventional microstrip antenna has been replaced by a conical patch with two shoulder angles are $48^{0}$ and $16^{\circ}$. On the surface of the conical patch, two reverse L-shaped are etched on the both edge side while a reverse T-shaped are formed on top edge of the patch. All slots are cut with width of $w_{s 1}$.

The second part is the coplanar waveguide (CPW) with feeder width of $w_{1}$ placed parallel to the ground plane with a gap of $d_{1}$. To ensure that the input impedance of antenna is equal $50 \Omega$, the CPW structure is coupled with a metallic rectangle plate, which has width of $l_{d}$, on the bottom layer of the dielectric substrate. Finally, two meandered lines width $w_{k}$ are placed parallelly to the vertical edge of the antenna. One terminal of this strip is connected to the ground plane while the other one is coupled with the edge of the antenna with a gap of $w_{c}$ via the triangle plate. 


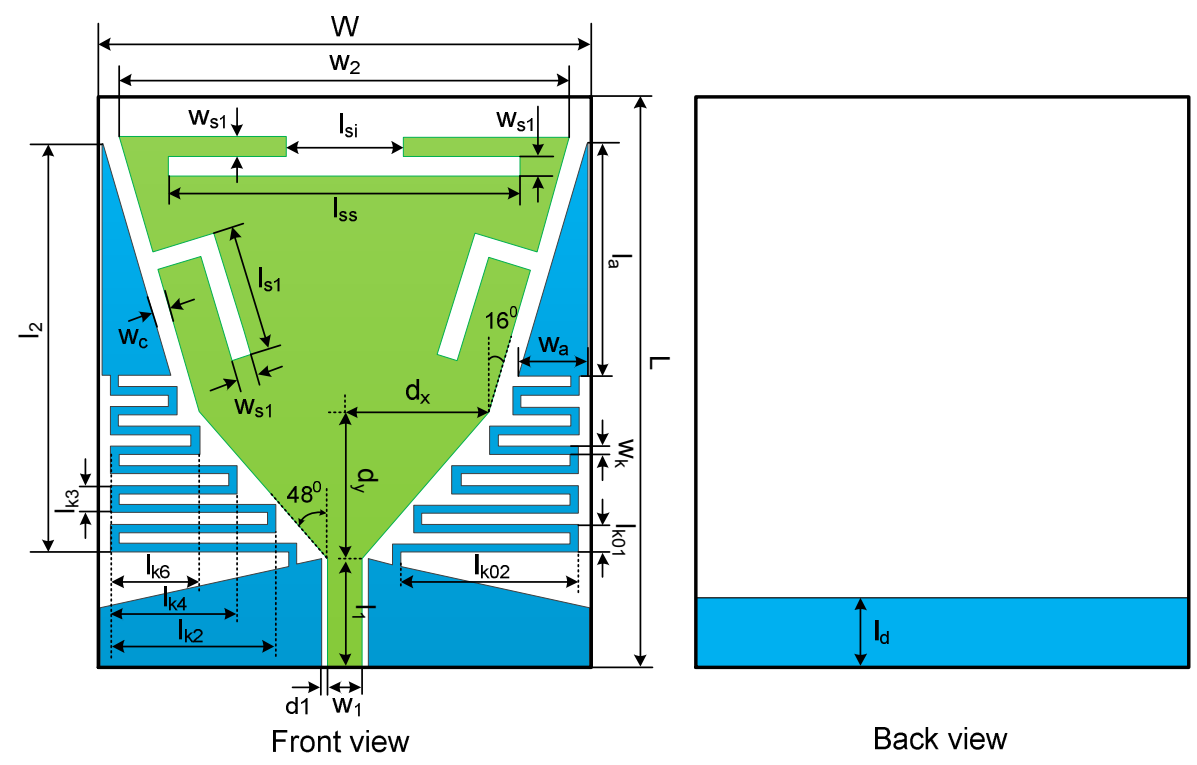

Figure 1. Configuration of proposed dual-broadband antenna.

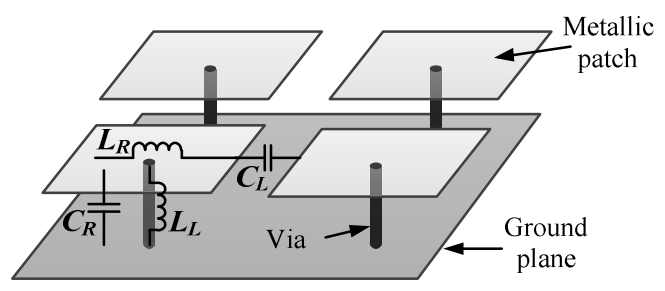

(a)

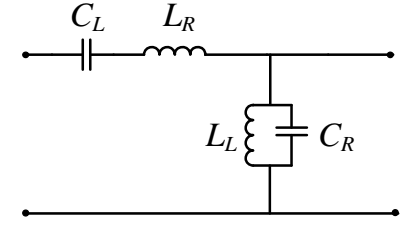

(b)

Figure 2. Model of CRLH-TL: (a) Mushroom-like EBG [18], and (b) Equivalent circuit.

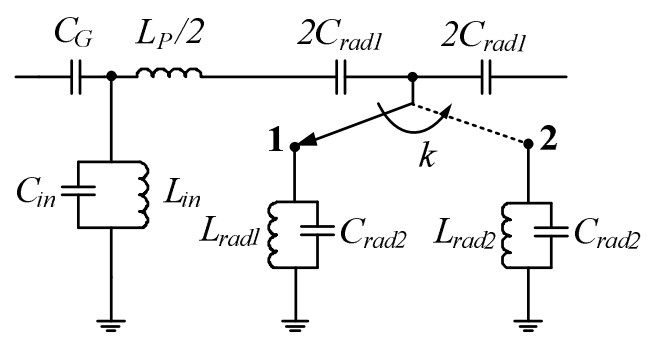

Figure 3. Equivalent circuit of proposed antenna.

Figure 2 illustrates the model and the equivalent circuit diagram of the conventional mushroom-like CRLH transmission line. In this diagram, the left-handed (LH) capacitance $C_{L}$ is created by gaps between the adjacent metallic plates on the top layer, while the LH inductance $L_{L}$ is produced by current flows from the metal plate to the metallic ground on the bottom layer through the metallic via. The use of metallic vias in the conventional CRLH structure is required to create LH inductance components, however, this leads to an inherent drawback of this structure is difficult to manufacture and influence the accuracy of the experimental results. Therefore, the design concept proposed in the metamaterial antenna is to convert conventional 
CRLH structure into the vialess structure. On the other hand, the gradual transform is used to enlarge two operating frequency bands of the proposed antenna.

The model proposed antenna is represented as the equivalent circuit diagram shown in Figure 3. From this figure, the capacitance $C_{G}$ is formed by the between the feeder on the top and the metallic patch on the bottom layer. The capacitance $C_{i n}$ is created by the gap between the feeder and the metallic ground plane while the ground plane is represented by the inductance $L_{\text {in }}$. These two components will be equivalent to the filter structure of transverse electromagnetic (TEM) wave mode at the input of the antenna. Capacitance $C_{\text {rad } 1}$ is made by inverse L-shaped slot cut at the edge side and the inverse T-shaped slit on the horizontal edge of the patch, while the meandered line is represented by the inductance $L_{\text {rad } 1}$. Capacitance $C_{\text {rad } 2}$ is made by the gap of the central patch and the extended area (triangle area) of the meandered line. In this design, the meandered line is represented by inductance $L_{\text {rad1 } 1}$ in case of low resonant frequency band and by inductance $L_{\text {rad } 2}$ when the antenna operates at a high frequency range. This happens due to the fringing effect that changes the role of meandered line. This change will be discussed in detail in the next section.

\subsection{Theorical calculation}

This section presents some basic calculations of the resonant frequency of the antenna in two frequency bands corresponding to the center frequency of $2.45 \mathrm{GHz}$ and $5.5 \mathrm{GHz}$ based on the equivalent circuit diagram shown in Figure 3. The dual-band antenna presented in Figure 1 is designed based on two basic principles: the structure of CLH-TL and fringing effects of metamaterials.

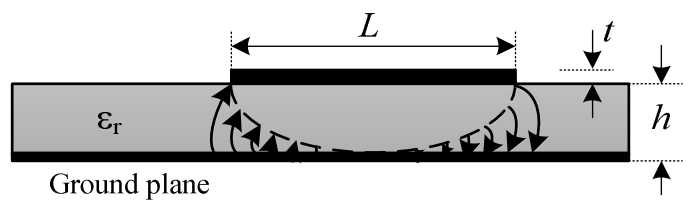

Figure 4. Fringing effect of metamaterials.

Figure 4 depicts the fringing effect of metamaterial. To simplify, the current distribution at the outer edge of the surface in the dielectric will lead to the increase of the electrical conductor length a range of $\Delta L$.

$$
L^{\prime}=L+\Delta L
$$

In which, $L$ is the physical length of the conductor.

$\Delta L$ is the extension length causes by the fringing effect of metamaterial.

$L^{\prime}$ is the electrical length of the conductor.

The extension length will alter the limited area of the wave modes in the waveguide. It can be calculated by the following formula:

$$
\Delta L=0.412 \times h \frac{\left(\varepsilon_{r e f f}+0.3\right)\left(\frac{W}{h}+0.264\right)}{\left(\varepsilon_{r e f f}-0.258\right)\left(\frac{W}{h}+0.8\right)}
$$

where, $W$ is the length corresponding to the $T E_{100}$ mode, which has the approximate value of $0.5 \lambda_{C}$, with $\lambda_{C}$ is wavelength in free space, $\varepsilon_{\text {reff }}$ is the effective dielectric constant, and $h$ is the thickness of the dielectric substrate. 


\subsubsection{At the frequency of $2.45 \mathrm{GHz}$}

In the equivalent model shown in Figure 3, the inductance $L_{\text {rad2 }}$ does not exist in the lower frequency range and the key $k$ is put at position 1. This can be explained in a simple way by fringing effect of metamaterial. As frequency increases, the length extension makes the physical size of $w_{1}$ increase compared to its actual size. A part of the current from this component flows through the narrow slit $d_{1}$ into the meandered line $l_{k i}$ forming imaging current on it. The value of $L_{\text {rad } 1}$ depends on electric field energy generated by the imaging current. Normally, this effect will be occurring when $d_{1}$ is equal $\Delta L$.

In this case, the value of $\Delta L$ is equal $0.2 \mathrm{~mm}$. We can see that the value of $\Delta L$ is greater than $d_{1}$, fringing effect occurred but it does not create the imaging current on the meandered line $l_{k i}$. This is because the difference between the length extension and the gap $d_{1}$ is not too large. Permeable area is not too big; the imaging current occurred with low amplitude and was attenuated during the propagation. As a result, equivalent circuit model of the antenna does not exist $C_{r a d 2}$ and $L_{r a d 2}$. Therefore, the resonant frequency of the antenna is calculated as follows:

$$
f_{C 1}=\frac{1}{2 \pi \sqrt{L_{\text {rad1 } 1}^{*} \cdot C_{\text {rad } 1}^{*}}}
$$

In which,

with

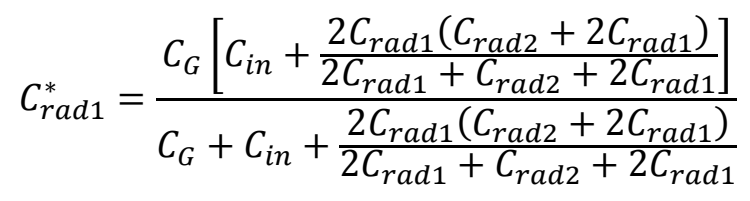

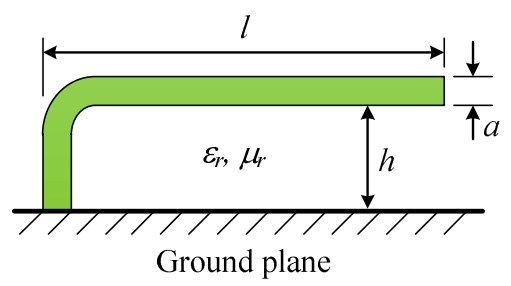

(a)

$$
\begin{gathered}
C_{\text {rad } 2}=\frac{\frac{\delta\left[\left(l_{s s}-l_{s i}\right)\left(w_{s 1}\right)\right]}{w_{s 1}} \cdot \frac{\delta\left(l_{s 1} \cdot w_{s 1}\right)}{w_{c}}}{\delta\left[\frac{\left(l_{s s}-l_{s i}\right)\left(w_{s 1}\right)}{w_{s 1}}+\frac{l_{s 1} \cdot w_{s 1}}{w_{c}}\right]} \\
L_{\text {rad } 1}^{*}=\frac{L_{\text {in }}\left(L_{\text {rad } 1}+L_{P / 2}\right)}{L_{\text {in }}+L_{\text {rad } 1}+L_{P / 2}}
\end{gathered}
$$

Figure 5. Calculation model of inductance value: (a) Inductance $L_{1}$, and (b) Inductance $L_{2}$.

Model of capacitance $L_{\text {rad } 1}$ is represented by two elements $L_{1}$ and $L_{2}$

$$
L_{\text {rad } 1}=L_{1}+L_{2}
$$


The inductance $L_{1}$ is generated due to the magnetic field energy stored between radiation plate which is fed directly (the component which has size of $l \times a$ shown in Figure 5 (a)) and meandered line $l_{k i}$ considered entirely as ground plane when fringing effect did not yet occur. While the equivalent inductance $L_{2}$ is created by the current distributed on the edge which is in contact with the dielectric and pairs of parallel strips $l_{k 2 i}$ and $l_{k(2 i+2)}$. From these equivalent models we can determine the value $L_{1}$ and $L_{2}$ as follows:

$$
\begin{aligned}
L_{1}=\sum_{1}^{15} \text { 0.0117. } l_{0} \cdot \mu_{r} \cdot \log _{10} & {\left[\frac{2 \cdot l_{k i}}{w_{k}}\left(\frac{l_{k i}+\sqrt{l_{k i}^{2}+w_{k}^{2}}}{l_{k i}+\sqrt{l_{k i}^{2}+4 h^{2}}}\right)\right] } \\
& + \text { 0.005. } \mu_{r} \cdot\left(\sqrt{l_{k i}^{2}+4 h^{2}}-\sqrt{l_{k i}^{2}+w_{k}^{2}}+\frac{1}{4}-2 \cdot h+w_{k}\right)
\end{aligned}
$$

where, $l_{0}$ is the edge length of the patch placed opposite the meandered line, and can be determined by the following formula:

$$
\begin{aligned}
& l_{0}=l_{1}+\frac{d_{y}}{\cos 48^{0}}+\frac{\left(l_{2}-l_{1}-d y\right)}{\cos 15^{0}} \\
& L_{2}=\frac{\mu_{0} \cdot \mu_{c c}}{\pi} \sum l_{k i} \cdot \cosh ^{-1} \cdot\left(\frac{\sum l_{k(2 i+1)}+\Delta L}{2 \cdot w_{k}}\right) \\
& =\frac{\mu_{0} \cdot \mu_{c c} \cdot \sum l_{k i}}{\pi}\left(\frac{\sum l_{k(2 i+1)}}{2 \cdot w_{k}}+\sqrt{\frac{\sum l_{k(2 i+1)}^{2}+\Delta L^{2}-1}{4 \cdot w_{k}^{2}}}\right)
\end{aligned}
$$

in which, $\mu_{c c}$ is the permeability of copper layer; $l_{k i}$ is the lengths formed the meandered line $(i=02,01,00,1,2 \ldots, 15) ; l_{k 2 i}$ is the length of the corresponding strips $l_{k 02} l_{k 00}, l_{k 2}, l_{k 4} \ldots, l_{k 14} ; l_{k(2 i+1)}$ is the length of the strips $l_{k 01}, l_{k 1}, l_{k 3} \ldots, l_{k 15}$.

\subsubsection{At the frequency of $5.5 \mathrm{GHz}$}

In this case, the value of $\Delta L$ is $0.262 \mathrm{~mm}$. The fringing effect occurs due to $\Delta L$ is greater than $d_{1}$, therefore, imaging current appeared on the meandered line $l_{k i}$ and turn it into a inductor with inductance $L_{\text {rad2 }}$ (the key $k$ is put at position 2). The resonant frequency is determined as follows:

where,

$$
f_{C 2}=\frac{1}{2 \pi \sqrt{L_{r a d 2}^{*} \cdot C_{r a d 2}^{*}}}
$$

$$
\begin{gathered}
C_{r a d 2}^{*}=\frac{C_{G}\left[C_{i n}+\frac{2 C_{r a d 1}\left(C_{r a d 2}+2 C_{r a d 1}\right)}{2 C_{r a d 1}+C_{r a d 2}+2 C_{r a d 1}}\right]}{C_{G}+C_{i n}+\frac{2 C_{r a d 1}\left(C_{r a d 2}+2 C_{r a d 1}\right)}{2 C_{r a d 1}+C_{r a d 2}+2 C_{r a d 1}}} \\
L_{r a d 2}^{*}=\frac{L_{i n}\left(L_{r a d 2}+L_{P / 2}\right)}{L_{i n}+L_{r a d 2}+L_{P / 2}} \\
L_{r a d 2}=\frac{0.8 \cdot w_{k}^{2} \cdot N^{2} \cdot \mu_{r}}{6 \cdot w_{k}+9 \cdot l_{k(2 i+1)}+10 l_{k 2 i}}
\end{gathered}
$$


where, $N$ is number of meander created by the strips $l_{k i}$, and it corresponds to the spirals of a conventional inductor.

\section{RESULTS AND DISCUSSIONS}

Firstly, the investigation on the impact of parametric size to the resonant frequency is considered to select the optimized parameters and meet the desired operational frequency range for the antenna. The investigation of size parameter is done with each parameter in turn, while the remaining parameters are fixed.

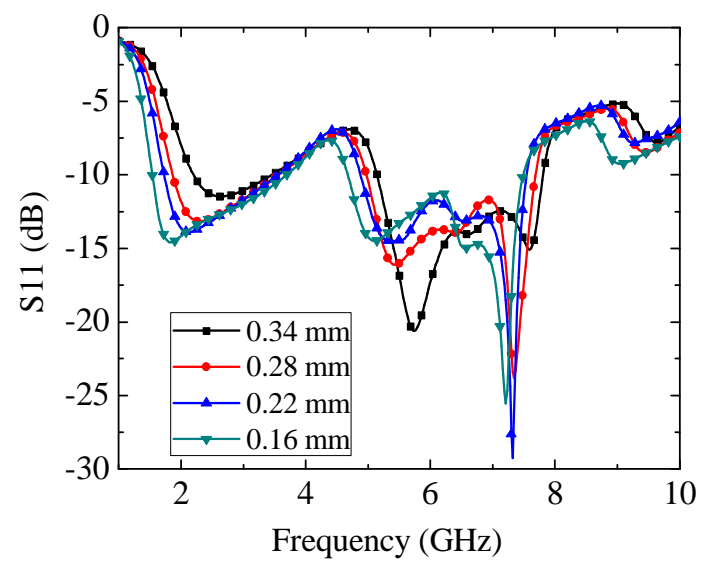

(a)

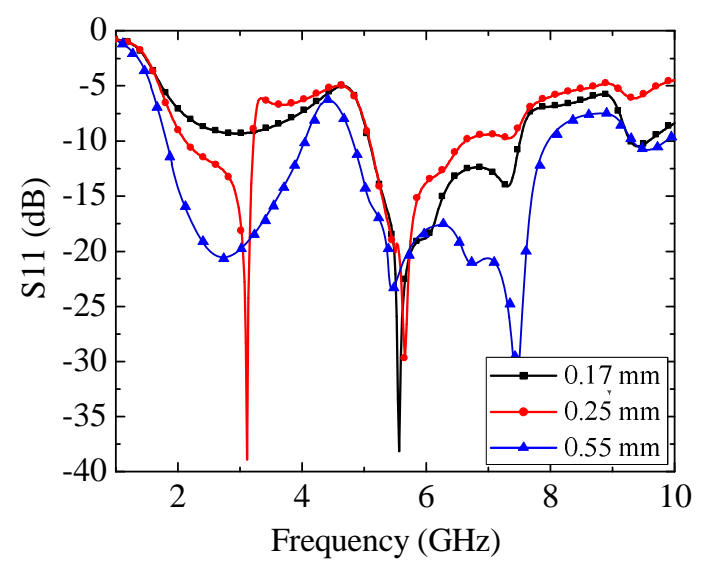

(b)

Figure 6. Simulated S11 of proposed antenna with different value of (a) width of meandered line $w_{k}$, and (b) gap between meandered line and patch $w_{c}$.

Figure 6 shows the simulated reflection coefficient S11 with different values of width $w_{k}$ of the meandered line. As observed in Figure 6(a), both resonance frequencies are shifted to the lower frequency range when the width $w_{k}$ reduced. We can see that the reduction of width $w_{k}$ leads to the increase of the meandered electrical length, and therefore making the increase of its equivalent inductance value. As discussed above, due to the impact of fringing effects that this value of meandered line at the frequency of $2.4 \mathrm{GHz}$ or $5.5 \mathrm{GHz}$ will be named $L_{\text {rad } 1}$ or $L_{\text {rad2 }}$, respectively. So, when we reduce the value of width $w_{k}$, the inductance $L_{\text {rad1 } 1}$ and $L_{\text {rad2 }}$ will be increased and make the two resonant frequency ranges of the antenna reduced in accordance with the formula (3) and (11).

Next, the simulated S11 results with the different values of the gap $w_{c}$ between the meandered line and central patch are shown in Figure 6(b). We can see that the bandwidths of both frequency ranges of the antenna are narrowed when $w_{c}$ reduced. On the other hand, the reflection coefficient of the antenna at $w_{c}=1.7 \mathrm{~mm}$ shows that the antenna only resonates at high frequency range. This is due to the loss of dual-band impedance matching of Chebyshev. The value of $w_{c}$ changes (such as the formula (13)) leading to the change of the reflection coefficient at high frequency band. Depending on how fast the change in value of $w_{c}$, the remaining band which does not depend on $w_{c}$ will also shrink, or the antenna will be mismatched because of the small value of the antenna impedance. 


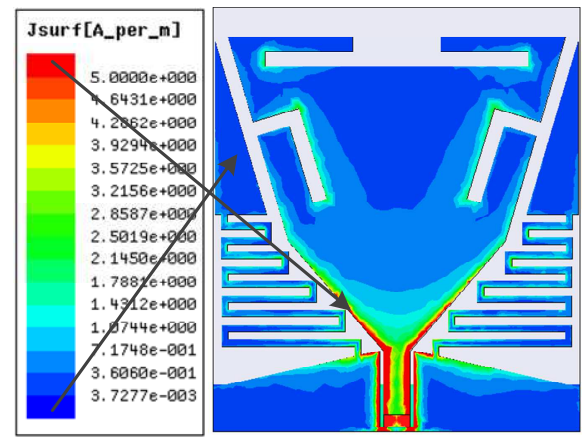

(a)

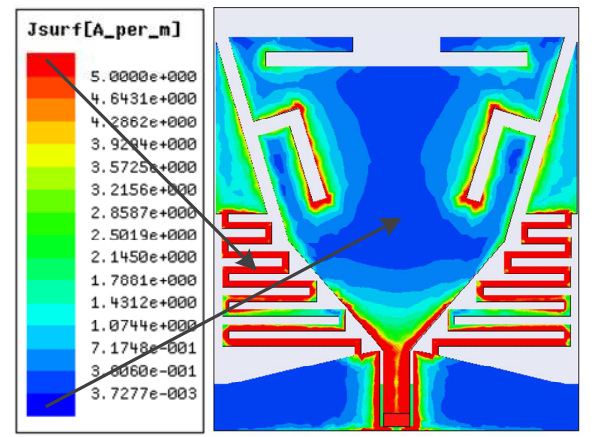

(b)

Figure 7. Current distribution of antenna with dielectric thickness of $0.8 \mathrm{~mm}$ at: (a) $2.45 \mathrm{GHz}$, and (b) $5.5 \mathrm{GHz}$.

Current distribution of the antenna at 2.45 and $5.5 \mathrm{GHz}$ are presented in Figure 7. It can be seen in Figure 7(a) that the current density at $2.45 \mathrm{GHz}$ frequency focuses mainly on the border of two edges and on a part of inverse L-shaped slits of the antenna. At this frequency, fringing effect occurs but not significantly (as analyzed in Section 2.1), and there is not current distribution on the meandered lines. However, the simulated result at $5.5 \mathrm{GHz}$ in Figure 7(b) shows that the current distribution is concentrated on the meandered line because of the influence of fringing effect. Besides, this effect also generates current distribution at the gap between the meandered line and the edges of the patch as well as at the inverse L- and T-shaped slits on the patch.

Table 1. Optimized parameter of proposed antenna (Unit: $\mathrm{mm}$ ).

\begin{tabular}{|c|c|c|c|c|c|}
\hline $\mathrm{W}$ & 16 & $\mathrm{w}_{\mathrm{k}}$ & 0.28 & $\mathrm{l}_{\mathrm{si}}$ & 3.75 \\
\hline $\mathrm{L}$ & 18 & $\mathrm{l}_{\mathrm{k} 01}$ & 0.91 & $\mathrm{l}_{\mathrm{ss}}$ & 11.25 \\
\hline $\mathrm{w}_{1}$ & 1.1 & $\mathrm{l}_{\mathrm{k} 02}$ & 5.67 & $\mathrm{~d}_{\mathrm{x}}$ & 4.06 \\
\hline $\mathrm{l}_{1}$ & 3.44 & $\mathrm{l}_{\mathrm{k} 2}$ & 5.38 & $\mathrm{~d}_{\mathrm{y}}$ & 4.69 \\
\hline $\mathrm{W}_{2}$ & 14.4 & $\mathrm{l}_{\mathrm{k} 3}$ & 0.88 & $\mathrm{w}_{\mathrm{c}}$ & 0.55 \\
\hline $\mathrm{l}_{2}$ & 13.4 & $\mathrm{l}_{\mathrm{k} 4}$ & 4.03 & $\mathrm{w}_{\mathrm{a}}$ & 2.19 \\
\hline $\mathrm{w}_{\mathrm{s} 1}$ & 0.63 & $\mathrm{l}_{\mathrm{k} 6}$ & 2.88 & $\mathrm{l}_{\mathrm{a}}$ & 7.36 \\
\hline $\mathrm{l}_{\mathrm{s} 1}$ & 4 & $\mathrm{~d}_{1}$ & 0.18 & & \\
\hline
\end{tabular}

The optimal size parameters of the proposed antenna are summarized in Table 1. These parameters are adjusted to achieve the operating frequency range that covering the WLAN system at 2.45 and $5.5 \mathrm{GHz}$ bands. The total size of the proposed antenna is $18 \mathrm{~mm} \times 16 \mathrm{~mm}$ (equivalent to $0,147 \lambda_{0} \times 0.13 \lambda_{0}$, with $\lambda_{0}$ is the wavelength in free space at frequency of 2.45 $\mathrm{GHz})$.

Simulated result of reflection coefficient of proposed antenna with optimized parameters is shown in Figure 8. It can be observed that that the antenna resonates at two frequency bands with $-10 \mathrm{~dB}$ bandwidth of $(1.92$ - 3.87) $\mathrm{GHz}$ and (4.94 - 7.76) GHz. The operating frequency bands of proposed antenna cover entirely the frequency bands of WLAN system spreading from 2.40 to $2.48 \mathrm{GHz}$ and from 5.2 to $5.8 \mathrm{GHz}$. 


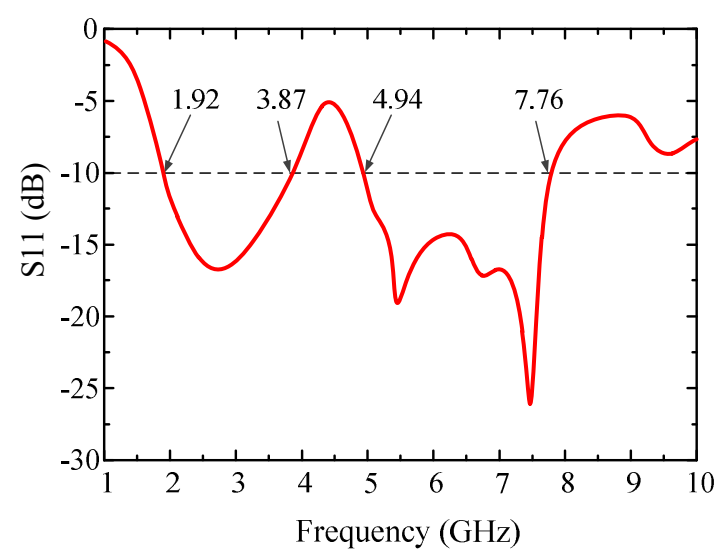

Figure 8. Simulated S11 of proposed antenna with dieclectric thickness of $0.8 \mathrm{~mm}$.

Next, we process the simulation of the proposed antenna with the dielectric thickness of 1.6 $\mathrm{mm}$ for evaluating the change of dielectric thickness to the fringing effects occurs in the proposed antenna at 2.45 and $5.5 \mathrm{GHz}$. The value of the increment length $\Delta L$ calculated at these both frequencies are 0.39 and $0.47 \mathrm{~mm}$, respectively.

For maintaining the input impedance at the feeder similar as the one of antenna with dielectric thickness of $0.8 \mathrm{~mm}$, the width of the feeder $w_{1}$ and the gap $d_{1}$ need to be adjusted. If we choice the value of $d_{1}$ is smaller than $\Delta L$, the fringing effects will occur at $2.45 \mathrm{GHz}$. However, in order to prove that the fringing effects at thickness of $1.6 \mathrm{~mm}$ will occur similar to the case of the dielectric thickness of $0.8 \mathrm{~mm}$, the value of $d_{1}$ is chosen as $0.385 \mathrm{~mm}$. Therefore, the width $w_{1}$ has a value of $1.68 \mathrm{~mm}$ in order to keep the input impedance of antenna stills equal $50 \Omega$.

At the frequency of $2.45 \mathrm{GHz}$, it can be seen that $\Delta L$ is a bit larger than $d_{1}$, then the fringing effects occurred but the image current did not appear on the meandered line. This is quite agreement with the current distribution simulation of the antenna with the thickness of 1.6 $\mathrm{mm}$ at $2.45 \mathrm{GHz}$ that is shown in Figure 9(a). However, the increment length of at $5.5 \mathrm{GHz}(0.47$ $\mathrm{mm}$ ) is significantly larger than the value of $d_{1}$, this will lead to the appearance of the image current on the meandered line due to the fringing effects. This is aslo consistent with the current distribution on the antenna presented in Figure 9(b).

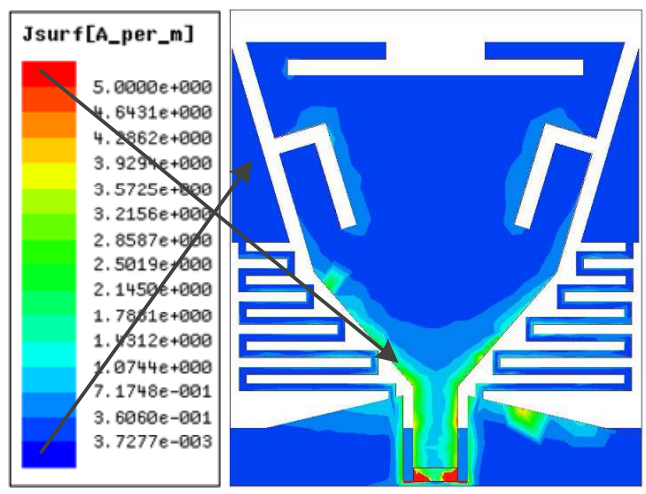

(a)

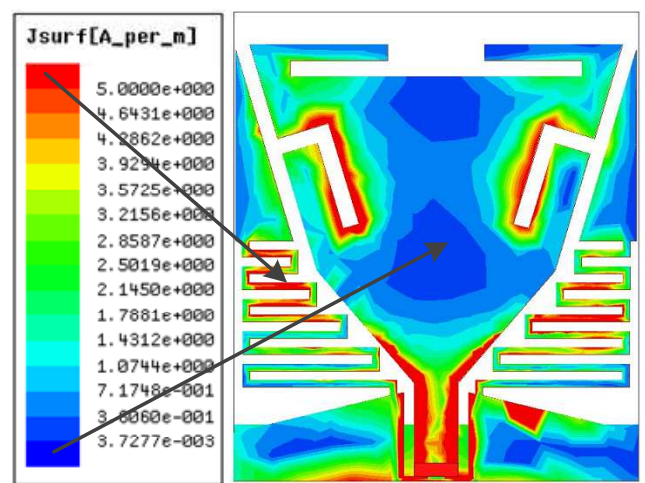

(b)

Figure 9. Current distribution of antenna with dielectric thickness of $1.6 \mathrm{~mm}$ at: (a) $2.45 \mathrm{GHz}$, and (b) $5.5 \mathrm{GHz}$. 
The simulation results of reflection coefficient of proposed antenna with $1.6 \mathrm{~mm}$ thickness substrate are shown in Figure 10. Observed in this figure, we can see that the two antenna resonating at the frequency range of 1.92 to $3.85 \mathrm{GHz}$ and 4.73 to $7.67 \mathrm{GHz}$, completely covered WLAN frequency range of 2.45 and $5.5 \mathrm{GHz}$.

Thus, in case of various thickness of dielectric substrate, fringing effects remained at the design frequency band if the value of $d_{1}$ and $w_{1}$ are appropriate chosen that still remain the input impedance of antenna.

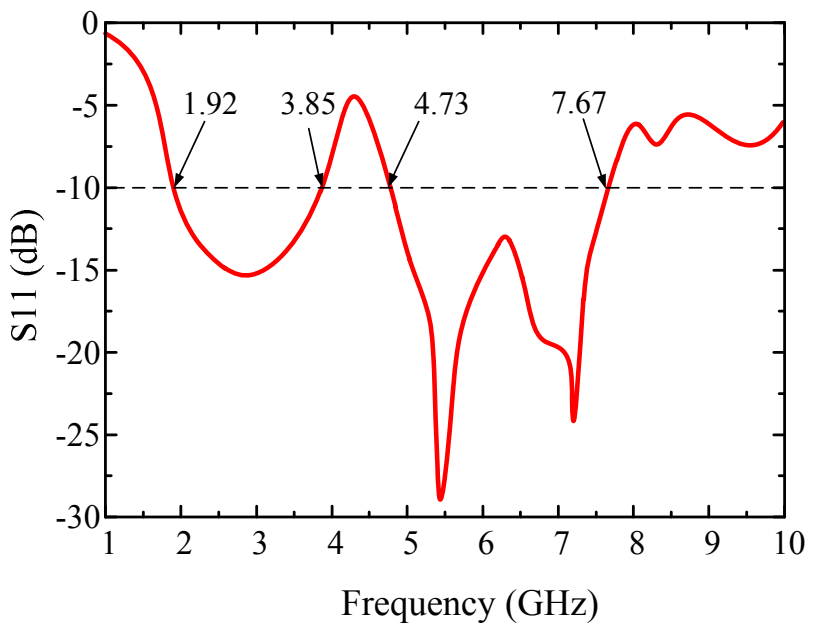

Figure 10. Simulated S11 of proposed antenna with dieclectric thickness of $1.6 \mathrm{~mm}$.

Radiation pattern simulated of the proposed antenna at the center frequency 2.45 and 5.5 $\mathrm{GHz}$ of WLAN system is presented in Figure 11. It is straightforward to recognize that the proposed antenna have an isotropic radiation pattern in $\mathrm{H}$ plane (YZ plane) at two above frequencies. The simulated gain of antenna at 2.45 and $5.5 \mathrm{GHz}$ are -0.43 and $1.23 \mathrm{~dB}$, respectively.

Table 2. Comparison with previous metamaterial antennas.

\begin{tabular}{|c|c|c|c|c|c|c|}
\hline \multirow{2}{*}{ References } & \multicolumn{2}{|c|}{ Dielectric } & \multirow{2}{*}{$\begin{array}{c}\text { Center } \\
\text { frequencies }\end{array}$} & \multirow{2}{*}{$\begin{array}{c}\text { Gain } \\
(\mathrm{dB})\end{array}$} & \multicolumn{2}{c|}{ Total size } \\
\cline { 7 - 7 } & $\varepsilon_{r}$ & $h$ & $(\mathrm{GHz})$ & & $(\mathrm{mm} \times \mathrm{mm})$ & $\left(\lambda_{0} \times \lambda_{0}\right)$ \\
\hline$[9]$ & 4.4 & 1.6 & $2.62 / 3.23$ & $1.1 / 1.7$ & $25 \times 20$ & $0.218 \times 0.175$ \\
\hline$[19]$ & 4.4 & 1.6 & $1.72 / 3$ & $1.2 / 1.5$ & $60 \times 50$ & $0.364 \times 0.287$ \\
\hline$[20]$ & 4.4 & 1.6 & $2.62 / 3.7$ & $0.9 / 1.9$ & $31.7 \times 27$ & $0.27 \times 0.235$ \\
\hline$[21]$ & 4.4 & 1.6 & $3.5 / 5.2$ & - & $26 \times 23$ & $0.3 \times 0.27$ \\
\hline$[22]$ & 4.4 & 0.8 & $2.4 / 5.8$ & $1.12 / 3.9$ & $27 \times 27$ & $0.216 \times 0.216$ \\
\hline$[23]$ & 4.4 & 0.8 & $2.6 / 5.2$ & $4.95 / 4.42$ & $34 \times 15$ & $0.29 \times 0.13$ \\
\hline \multirow{2}{*}{ This work } & 4.4 & 0.8 & $2.45 / 5.5$ & $-0.43 / 1.23$ & $18 \times 16$ & $0.147 \times 0.13$ \\
& 4.4 & 1.6 & $2.45 / 5.5$ & $-0.33 / 1.27$ & $18 \times 16$ & $0.147 \times 0.13$ \\
\hline
\end{tabular}


Table 2 provides a comparison in terms of total size between the proposed antennas and metamaterial antennas that have been reported recently. All the reference models are dual-band antenna. Overall dimensions of the antennas are compared in units of millimeter and the wavelength in free-space at low frequency of antenna. It is clearly evident that the proposed filter has a smaller size than the one of listed previous works. However, the gain of proposed antenna at the lower frequency is much smaller than the ones of reference antennas.

In order to validate the proposed model, optimized antenna is fabricated and experimentally

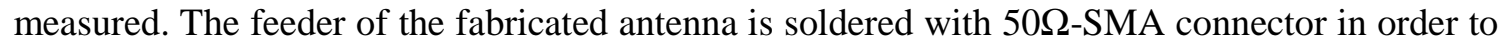
measure the scatter parameters. The measurement is performed by the PNA-X Keysight Network Analyzer with measured range from $125 \mathrm{MHz}$ to $26.5 \mathrm{GHz}$. The fabricated prototype is shown in Figure 12, while the S11 measured result of antenna is presented in Figure 13. The measured $-10 \mathrm{~dB}$ bandwidth of antenna shows that it can operate at two dual-broadband in which the lower band ranges from 1.87 to $3.62 \mathrm{GHz}$ while the higher one covers a frequency band of $(4.85-7.52) \mathrm{GHz}$.

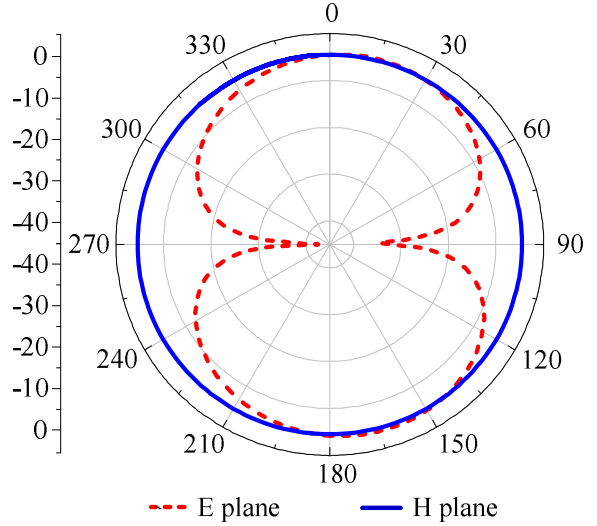

(a)

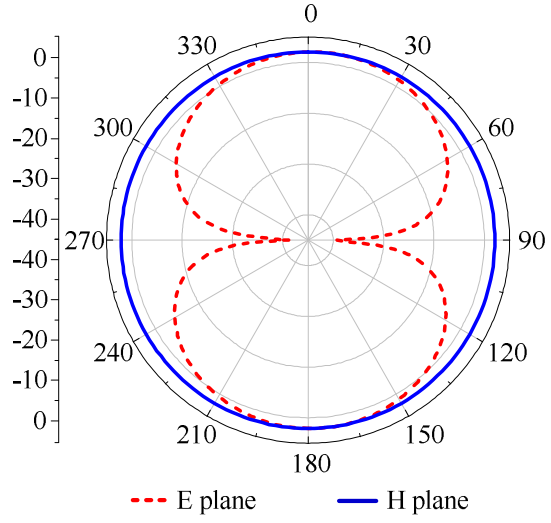

(b)

Figure 11. Radiation pattern of proposed antenna at: (a) $2.45 \mathrm{GHz}$, and (b) $5.5 \mathrm{GHz}$.

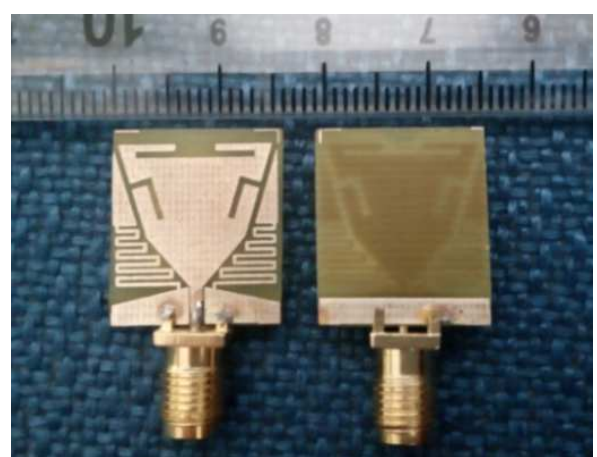

Figure 12. Prototype of proposed antenna.

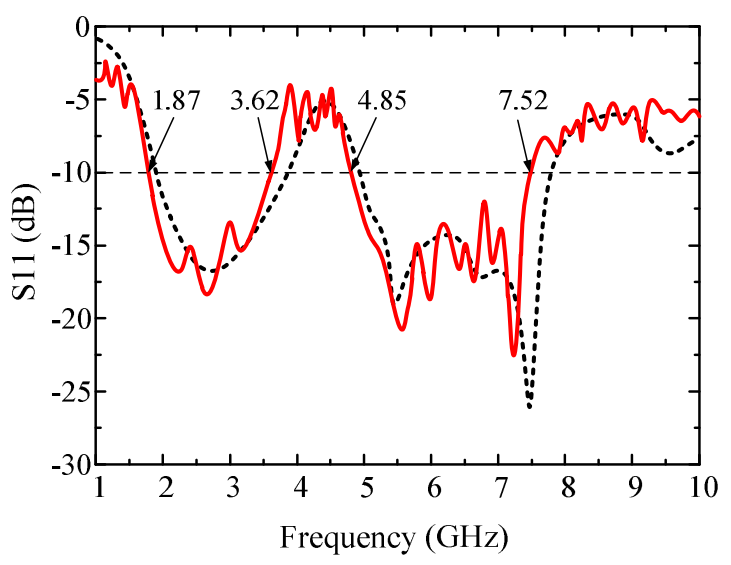

Figure 13. Measured S11 of fabricated antenna.

\section{CONCLUSIONS}

A compact dual-broadband metamaterial antenna has been designed, fabricated and measured. The proposed antenna used composite right-left handed transmission line (CRLH-TL) 
for size reduction, combined with fringing effects of metamaterial to achieve dual frequency bands. Gradual transform was employed to broaden the bandwidth of both operation bands of the antenna. It was demonstrated that the proposed antenna has dual impedance bandwidths ranged from 1.87 to $3.62 \mathrm{GHz}$ in the lower frequency band and 4.85 to $7.72 \mathrm{GHz}$ in the higher frequency band that covered the WLAN frequency bands of $(2.40-2.485) \mathrm{GHz}$ and $(5.2-5.8)$ GHz. In both bands, the antenna can generate a good omnidirectional, monopole-like radiation pattern. With small size, vialess, easy to manufacture and low cost the proposed antenna can be a good candidate for applications of WLAN systems.

\section{REFERENCES}

1. Eleftheriades G. V., Iyer A. K., and P. C. Kremer P. C. - Planar negative refractive index media using periodically LC loaded transmission lines, IEEE Transactions on Microwave Theory and Techniques $\mathbf{5 0}$ (2002) 2702-2712.

2. Zhu J. and Eleftheriades G. V. - A compact transmission-line metamaterial antenna with extended bandwidth, IEEE Antennas and Wireless Propagation Letters 8 (2009) 295-298.

3. Reddy S. V., Sarkar D., Saurav K., and Srivastava K. V. - A compact CRLH unit cell loaded triple-band monopole antenna, Microwave and Optical Technology Letters 57 (2015) 115-119.

4. Lai A., Leong K. M., and Itoh T. - Infinite wavelength resonant antennas with monopolar radiation pattern based on periodic structures, IEEE Transactions on Antennas and Propagation 55 (2007) 868-876.

5. Rajeshkumar V. and Raghavan S. - A compact metamaterial inspired triple band antenna for reconfigurable WLAN/WiMAX applications, AEU-International Journal of Electronics and Communications 69 (2015) 274-280.

6. Zheng L., Wang G., and Geng L. - Compact coplanar waveguide (CPW)-fed tunable wideband resonant antennas using metamaterial transmission line, Progress In Electromagnetics Research Letters 40 (2013) 19-28.

7. Locatelli A., Modotto D., Angelis C., Boscolo S., Midrio M., and Capobianco A. D. Design of fully printed omnidirectional CRLH loop antennas for WLAN technology, Microwave and Optical Technology Letters 56 (2014) 1405-1408.

8. Bala B. D., Rahim M. K. A., Murad N. A., and Rahim S. K. A. - Dual band metamaterial antenna with loaded resonators, 2013 Asia-Pacific Microwave Conference Proceedings (APMC) (2013) 1151-1153.

9. Sharma S. K. and Chaudhary R. K. - Metamaterial inspired dual-band antenna with modified CSRR and EBG loading, 2015 IEEE International Symposium on Antennas and Propagation \& USNC/URSI National Radio Science Meeting, 2015, pp. 472-473.

10. Antoniades M. A. and Eleftheriades G. V. - A folded-monopole model for electrically small NRI-TL metamaterial antennas, IEEE Antennas and Wireless Propagation Letters 7 (2008) 425-428.

11. Zhu J., Antoniades M. A., and Eleftheriades G. V. - A compact tri-band monopole antenna with single-cell metamaterial loading, IEEE Transactions on Antennas and Propagation 58 (2010) 1031-1038. 
12. Ibrahim W. W., Abdalla M. A., Allam A., Mohamed A. A. N., and Elregaily H. A. - A compact and dual band metamaterial substrate integrated waveguide antenna, 2013 IEEE Antennas and Propagation Society International Symposium (APSURSI) (2013) 966-967.

13. Zhu J. and Eleftheriades G. - Dual-band metamaterial-inspired small monopole antenna for WiFi applications, Electronics Letters 45 (2009) 1104-1106.

14. Bala B., Rahim M., and Murad N. - Composite right/left-handed dual-band metamaterial antenna with improved gain and efficiency, Microwave and Optical Technology Letters 56 (2014) 1575-1579.

15. Ren W., Shi Z., Liu H., and Chen K. - Novel compact 2.4/5-GHz dual-band T-slot antenna for WLAN operations, Microwave and Optical Technology Letters 49 (2007) 1236-1238.

16. Wang J. and He X. - Analysis and design of a novel compact multiband printed monopole antenna, International Journal of Antennas and Propagation 2013 (2013).

17. Ren W. - Compact Dual-Band Slot Antenna for 2. 4/5ghz WLAN Applications, Progress In Electromagnetics Research B 8 (2008) 319-327.

18. Yang F. and Rahmat-Samii Y. - Electromagnetic band gap structures in antenna engineering: Cambridge university press Cambridge, 2009.

19. Sharma S. K., Gupta D., Mulchandani J. D., and Chaudhary R. K. - A dumbbell-shaped dual-band metamaterial antenna using FDTD technique, Progress In Electromagnetics Research Letters 56 (2015) 25-30.

20. Si L. M., Zhu W., and Sun H. J. - A compact, planar, and CPW-fed metamaterial-inspired dual-band antenna, IEEE Antennas and Wireless Propagation Letters 12 (2013) 305-308.

21. Abdalla M. A. - A dual mode CRLH TL metamaterial antenna, 2014 IEEE Antennas and Propagation Society International Symposium (APSURSI) (2014) 793-794.

22. Abioghli M. and Sadeghzadeh R. A. - A new compact dual-band bow-tie microstrip Antenna for WLAN applications, IETE Journal of Research 59 (2013) 693-697.

23. Lee Y. C. and Sun J. S. - Compact printed slot antennas for wireless dual-and multi-band operations, Progress In Electromagnetics Research 88 (2008) 289-305. 